\title{
Maternal protein restriction before pregnancy reduces offspring early body mass and affects glucose metabolism in C57BL/6JBom mice
}

Dudele, Anete; Lund, S.; Jessen, N.; Wegener, G. ; Winther, G. ; Elnif, Jan; Frische, Sebastian; Wang, Tobias; Mayntz, David

Published in:

Journal of Developmental Origins of Health and Disease

DOI:

$10.1017 / \mathrm{S} 2040174412000347$

Publication date:

2012

Document version

Early version, also known as pre-print

Citation for published version (APA):

Dudele, A., Lund, S., Jessen, N., Wegener, G., Winther, G., Elnif, J., Frische, S., Wang, T., \& Mayntz, D. (2012). Maternal protein restriction before pregnancy reduces offspring early body mass and affects glucose metabolism in C57BL/6JBom mice. Journal of Developmental Origins of Health and Disease, 3(5), 364-374.

https://doi.org/10.1017/S2040174412000347 


\title{
Maternal protein restriction before pregnancy reduces offspring early body mass and affects glucose metabolism in C57BL/6JBom mice
}

\author{
A. Dudele ${ }^{1 *}$, S. Lund $^{2}$, N. Jessen ${ }^{2}$, G. Wegener ${ }^{3}$, G. Winther ${ }^{3}$, J. Elnif ${ }^{4}$, S. Frische ${ }^{5}$, T. Wang ${ }^{1}$ and \\ D. Mayntz ${ }^{6,7}$ \\ ${ }^{1}$ Department of Bioscience, Zoophysiology, University of Aarhus, Aarhus C, Denmark \\ ${ }^{2}$ Medical Research Laboratory and Medical Department M (Endocrinology and Diabetes), Aarhus University Hospital, Aarhus C, \\ Denmark \\ ${ }^{3}$ Center for Basic Psychiatric Research, Aarhus University Hospital, Risskov, Denmark \\ ${ }^{4}$ Animal Nutrition, Department of Basic Animal and Veterinary Sciences, University of Copenhagen, Copenhagen, Denmark \\ ${ }^{5}$ The Water and Salt Research Centre, Institute of Anatomy, University of Aarhus, Aarhus C, Denmark \\ ${ }^{6}$ Department of Bioscience, Ecology and Genetics, University of Aarhus, Aarhus C, Denmark \\ ${ }^{7}$ Department of Genetics and Biotechnology, University of Aarhus, Research Centre Foulum, Tjele, Denmark
}

\begin{abstract}
Dietary protein restriction in pregnant females reduces offspring birth weight and increases the risk of developing obesity, type 2 diabetes and cardiovascular disease. Despite these grave consequences, few studies have addressed the effects of preconceptional maternal malnutrition. Here we investigate how a preconceptional low-protein (LP) diet affects offspring body mass and insulin-regulated glucose metabolism. Ten-week-old female mice (C57BL/6JBom) received either an LP or isocaloric control diet ( $8 \%$ and $22 \%$ crude protein, respectively) for 10 weeks before conception, but were thereafter fed standard laboratory chow (22.5\% crude protein) during pregnancy, lactation and offspring growth. When the offspring were 10 weeks old, they were subjected to an intraperitoneal glucose tolerance test (GTT), and sacrificed after a 5-day recovery period to determine visceral organ mass. Body mass of LP male offspring was significantly lower at weaning compared with controls. A similar, nonsignificant, tendency was observed for LP female offspring. These differences in body mass disappeared within 1 week after weaning, a consequence of catch-up growth in LP offspring. GTTs of 10-week-old offspring revealed enhanced insulin sensitivity in LP offspring of both sexes. No differences were found in body mass, food intake or absolute size of visceral organs of adult offspring. Our results indicate that maternal protein restriction imposed before pregnancy produces effects similar to postconceptional malnutrition, namely, low birth weight, catch-up growth and enhanced insulin sensitivity at young adulthood. This could imply an increased risk of offspring developing lifestyle-acquired diseases during adulthood.
\end{abstract}

Received 8 February 2012; Revised 5 April 2012; Accepted 27 April 2012

Key words: glucose tolerance, low-protein diet, preconception nutrition

\section{Introduction}

Non-communicable diseases, such as cardiovascular disease (CVD), type II diabetes mellitus (T2DM), cancer and chronic respiratory diseases, are responsible for a large proportion of early deaths worldwide. ${ }^{1}$ Although certain lifestyle choices in combination with genetic predisposition are recognized to markedly increase the risk of developing these diseases, ${ }^{2}$ the physiological and nutritional state of the mother during gestation and lactation also have a significant role in determining future health of the offspring. ${ }^{3,4}$

It was highly controversial when Barker proposed the concept of 'Fetal origins of adult disease' in 1990,5 but epidemiological studies revealed a clear relationship between

*Address for correspondence: A. Dudele, M.Sc., Department of Bioscience, Zoophysiology, University of Aarhus, C.F. Møllers allé, Building 1131, DK-8000, Aarhus C, Denmark.

(Email a.dudele@gmail.com) maternal undernutrition during pregnancy and postnatal exposure to nutrient-rich environment, with an increased prevalence of CVD and T2DM in adult male offspring. ${ }^{6,7}$ These observations lead to the formation of thrifty phenotype hypothesis ${ }^{8}$ that has been widely accepted in the present time. In addition to epidemiological observations, animal models have repeatedly shown that intrauterine growth restriction imposed by maternal protein or caloric restriction during pregnancy leads to low birth weight and development of CVD, T2DM and obesity in the offspring. ${ }^{9,10}$

Although the evidence appears strong and is consistent when it comes to effects caused by maternal nutrition during pregnancy, the effects of maternal nutrition before pregnancy on the future offspring are far less studied. Although indistinct, epidemiological evidence indicates an association of preconceptional consumption of protein and vegetables with reduced risk for low birth weight ${ }^{11,12}$ and consecutively development of T2DM and CVD. ${ }^{13,14}$ Animal studies supplement these 
findings by demonstrating that maternal caloric and protein restriction exclusively before pregnancy leads to accelerated postnatal growth in ewes and affects offspring vascular function in ewes and mice, ${ }^{15,16}$ as well as increases male offspring adiposity at young adulthood in mice. ${ }^{17}$

Here we study how reduced maternal protein intake before conception affects offspring early body mass and their insulin-mediated glucose metabolism. As the offspring are not directly exposed to protein restriction during development, we expect the effects to operate through maternal body composition and/or physiological state, and we therefore examine maternal food intake, body mass, organ size and adiposity before conception to investigate whether any of these factors account for changes induced in the offspring.

\section{Materials and Methods}

\section{Animals and housing}

All experiments were performed in accordance with the principles and guidelines of Danish legislation on animal welfare, permit number 2006/561-1257. Danish Inspectorate for Animal Experiments specifically approved this study.

Eight-week-old virgin C57BL/6JBom mice (53 females and 36 males) were obtained from Taconic (Lille Skensved, Denmark) and caged individually in type 2 Macrolon cages (length: $23 \mathrm{~cm}$; width: $17 \mathrm{~cm}$; height: $14 \mathrm{~cm}$ ) at $12: 12 \mathrm{~h}$ light:dark regime, $23^{\circ} \mathrm{C}$ and $60 \%$ humidity. Food and water was available ad libitum at all times, except for the overnight starvation before Glucose Tolerance Test (GTT), when food was removed for $14 \mathrm{~h}$.

\section{Experimental diets}

Four dry pellet diets were used in the experiment (composition is described in Table 1). NIH \#31 $\mathrm{M}$ rodent diet was purchased from Taconic (Denmark) and two custom-made isocaloric experimental diets - Low protein (LP) and Standard (ST) were purchased from Brogaarden/Altromin (Denmark). Both diets were similar in composition apart from the crude protein and carbohydrate content (Table 1). Laboratory chow diet (Altromin 1310) for rats and mice (Brogaarden/Altromin, Denmark) was used as a standard laboratory chow diet.

\section{Experimental protocol}

Before the start of the experiment, all 53 female mice were allowed to acclimate to the facilities for 2 weeks; during this period, they were fed the same diet as they received at the breeding facilities (NIH \#31 M rodent diet). Subsequently, they were weighed and divided into two groups that received either an LP $(n=27)$ or an ST $(n=26)$ diet for the next 9-10 weeks. Following that, nine mice from each treatment were subjected to a GTT, allowed to recover from this test for 5 days and sacrificed by cervical dislocation. The remaining mice $\left(n=35, n_{\mathrm{ST}}=17 ; n_{\mathrm{LP}}=18\right)$ were placed in cages, with an 8-9-week-old male for every female, for $48 \mathrm{~h}$. From this point onwards, all animals received laboratory chow diet (Altromin 1310). After mating, the male was removed, but the female remained in the individual cage until offspring were born and weaned onto laboratory chow diet (Altromin 1310) at the age of 23 days and caged individually. Laboratory chow diet (Altromin 1310) was used after mating and thereafter to ensure that both groups of female mice were exposed to a change in the diet close to the time of conception. In order to minimize handling stress upon the animals, neither maternal nor offspring body masses were measured at birth. Owing to the low litter numbers, litter sizes were not adjusted. At the age of 10 weeks, the offspring were subjected to GTT, allowed to recover from it for 5 days and sacrificed by cervical dislocation (Fig. 1).

\section{Food intake measurements}

Maternal food intake was measured weekly using a spillage collecting system, for example, ${ }^{18}$ on individually caged animals. Animals received a preweighed portion of food, large enough to ensure that they had access to food at all times. After 1 week, the food remaining in the feeding tray and spillage collector was collected and stored at $-25^{\circ} \mathrm{C}$ for later measurements. Before the measurements, the collected food was defrosted and dried at $60^{\circ} \mathrm{C}$ for $24 \mathrm{~h}$, and weighed to the nearest $0.001 \mathrm{~g}$. A standard curve for conversion of wet mass to dry mass was created for each feed separately.

Offspring food intake was measured on individually housed animals once a week after weaning. As all offspring received

Table 1. Composition of the diets used before and during the experiment

\begin{tabular}{lcccc}
\hline Diet & NIH \#31 M rodent diet & LP & ST & Laboratory chow diet (Altromin 1310) \\
\hline Crude protein & 18 & 8.4 & 21.5 & 22.5 \\
Carbohydrates & 53 & 60 & 47.2 & 50.5 \\
Crude fat & 5.3 & 7.2 & 7.2 & 5.0 \\
Crude fiber & 4.5 & 5.0 & 5.1 & 4.5 \\
Energy (kcal/g) & 3.4 & 3.3 & 3.3 & 2.99 \\
\hline
\end{tabular}

LP, low protein; ST, standard diet.

Values presented as g per $100 \mathrm{~g}$ dry mass. 


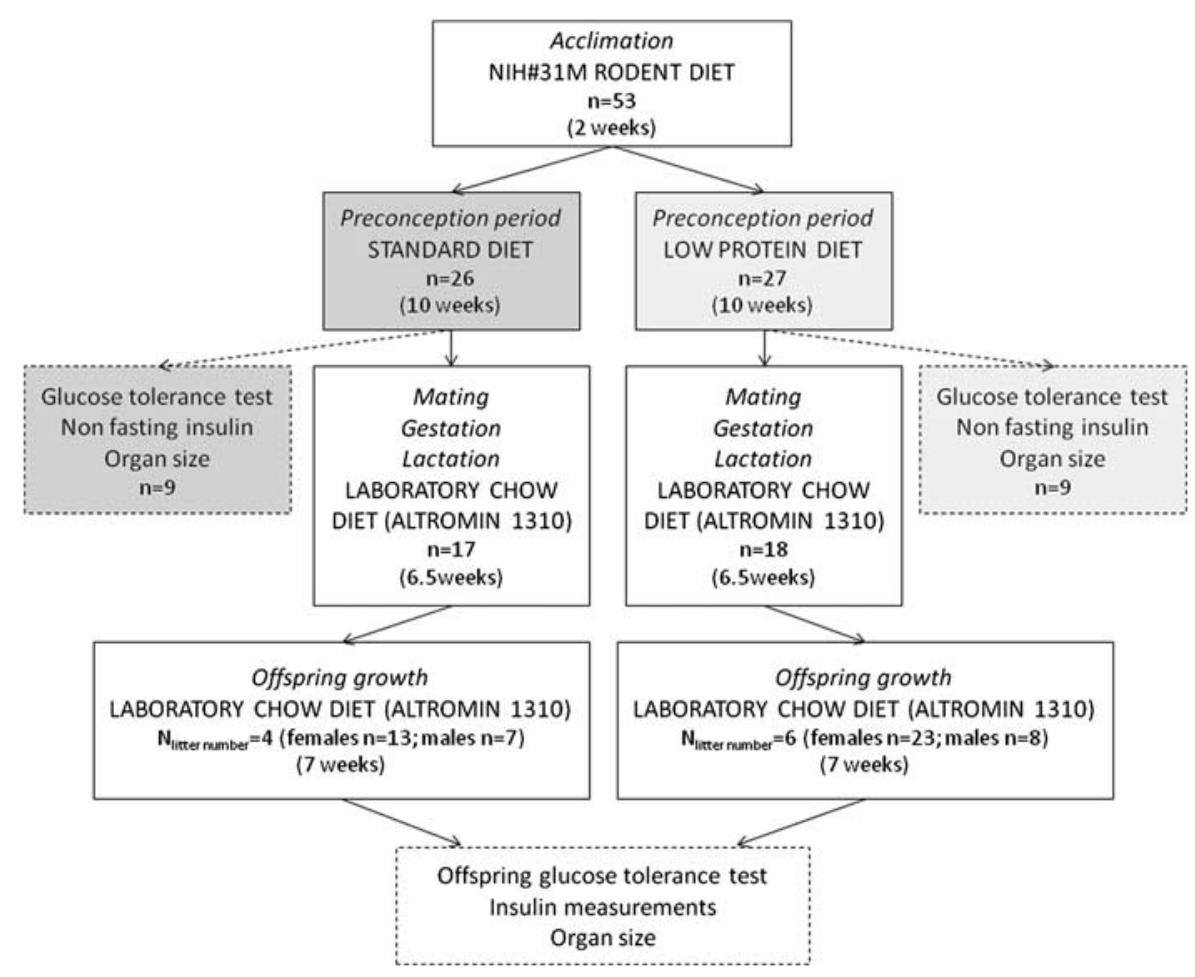

Fig. 1. Experimental protocol used during the study.

laboratory chow diet (Altromin 1310), spillage of food was expected to be similar between groups, and therefore only the difference of given and leftover food in the feeding tray was measured.

\section{GTTs}

GTTs were performed after a 14-h overnight fast. Upon measuring fasting blood glucose, $10 \%$ glucose solution was injected intraperitoneally $(2 \mathrm{~g} / \mathrm{kg})$, and blood was collected from an incision at the tip of the tail for determination of glucose concentration at 15, 30, 60 and $120 \mathrm{~min}$. All blood glucose concentration measurements were performed using Bayer Contour ${ }^{\circledR}$ glucometer and Bayer Contour ${ }^{\circledR}$ test strips.

\section{Insulin levels}

Blood samples for insulin measurements were collected after the $14 \mathrm{~h}$ starvation preceding the GTT and $2 \mathrm{~h}$ after glucose injection during GTT. Blood was collected from an incision at the tip of the tail into a heparinized hematoctit tube. Trunk blood was collected after sacrifice to determine non-fasting insulin concentration. All collected blood samples were heparinized and spun, and plasma was collected and frozen at $-80^{\circ} \mathrm{C}$ for later analysis. Plasma insulin concentration was measured using Ultra Sensitive Mouse Insulin ELISA kit according to manufacturer's instructions.

\section{Organ size measurements}

Five days after GTT, animals were weighed to a precision of $0.1 \mathrm{~g}$ and sacrificed by cervical dislocation and decapitation. Kidneys, liver and heart were dissected, blotted on a filter paper in a standardized manner and weighed. The gastrointestinal tract was dissected free to determine the length from the pyloric sphincter to the rectum and the length of the small intestine with precision to $1 \mathrm{~mm}$. Stomach, cecum and colon were separated and flushed with $0.9 \%$ saline, dried at $60^{\circ} \mathrm{C}$ for $72 \mathrm{~h}$ and weighed. The small intestine was blotted on filter paper and wet mass taken. The mucosal layer was separated from a 4-6-cm-long piece of the small intestine (as described by Jensen et $a l^{19}{ }^{19}$. The proportion of mucosal layer to the layer of muscular tissue of the small intestine was determined after drying both layers separately for $72 \mathrm{~h}$ at $60^{\circ} \mathrm{C}$. The rest of the small intestine was used for another experiment, and therefore wet mass to dry mass relationship of the 4-6-cm-long piece was used to calculate the dry mass of the whole small intestine. All weight measurements were recorded with precision to $0.1 \mathrm{mg}$.

\section{Data analysis}

Data are presented as means $( \pm$ S.E. $)$ or as least square means ( \pm S.E.), and the differences between treatment groups (ST $v$. LP) were tested within the same sex by Student's $t$-test. For comparison of GTT results, the area under the GTT curve [Area Under the Curve (AUC)] was calculated. The fasting 
blood glucose concentration was compared between ST and LP groups within the same gender by Student's $t$-test, and if the values were not significantly different - which was always the case - baseline was calculated (fasting glucose value $X$ $120 \mathrm{~min}$ ) and subtracted from the total AUC.

Analysis of Covariance (ANCOVA) was used to test the effects of body mass and dietary treatment on organ size of females and the effects of maternal body mass and diet before conception on offspring early body mass. Maternal diet and offspring body mass were used as covariates when offspring organ size was measured. ANCOVA was used to test the effect of maternal body mass before conception on mean body mass of male or female offspring per litter at weaning. Maternal diet and litter size were included as covariates initially, but were removed from the model if not significant.

Litter size was always included in the covariate model initially, but was removed if nonsignificant, which was always the case.

Offspring growth and food intake were compared using repeated measures Multivariate Analysis of Variance (MANOVA) in both genders separately.

Homogeneity of variances was tested using Levene's test $(\alpha>0.05)$, and if the variances proved to be unequal data were compared using Welch Analysis of Variance (ANOVA).

All data analysis was conducted using JMP 8 and Sigma Plot 11.0.

\section{Results}

\section{Food intake and body mass changes of mothers}

After 9 weeks of feeding on experimental diets, the total food consumption of females averaged $149 \pm 2 \mathrm{~g}$ (dry mass) per animal in both groups, and there was no significant difference between LP and ST treatments ( $t$-test, $\mathrm{DF}=51, t=0.08$, $P=0.94)$. On average, after 9 weeks of feeding dams had consumed a total of $493 \pm 5 \mathrm{kcal}$ per animal and this result was not affected by the dietary treatment $(t$-test, $\mathrm{DF}=51, t=0.08$, $P=0.94)$. However, owing to differences in the protein content of experimental diets, over 9 weeks of feeding females on the LP diet ingested a total of $12.5 \pm 0.4 \mathrm{~g}$ crude protein, whereas females on the ST diet consumed 2.6 times more protein $(32.1 \pm 0.4 \mathrm{~g} ; t$-test, $\mathrm{DF}=51, t=39.64, P<0.0001)$.

At the end of the feeding period, we observed significant differences in female body mass. Dams exposed to the LP diet had lower body mass and weighed $21.7 \pm 0.3 \mathrm{~g}$, whereas ST females weighed $22.5 \pm 0.3 \mathrm{~g}$ ( $t$-test, $\mathrm{DF}=51, t=2.1, P=0.04)$.

We found no significant differences in the body mass of dams exposed to experimental diets and used for GTT and organ size measurements (Table 2).

\section{GTT and non-fasting plasma insulin concentration of females fed on experimental diets}

We found no significant differences in GTT results (Fig. 2; $t$-test, $\mathrm{DF}=15, t=-0.71, P=0.49)$ between females fed the LP diet and those fed the ST diet for 9 weeks. However, we observed a nearly significant reduction in the non-fasting plasma insulin concentration of females fed the LP diet $(0.33 \pm 0.07 \mu \mathrm{g} / \mathrm{l} ; n=8)$ compared with the non-fasting insulin levels of females fed the ST diet $(0.66 \pm 0.14 \mu \mathrm{g} / \mathrm{l}$; $n=8 ; t$-test, $\mathrm{DF}=14, t=2.06, P=0.06$ ).

\section{Organ sizes of females fed experimental diets}

Females fed the ST diet had significantly larger kidneys than females on the LP diet (Table 2). The difference remained

Table 2. Organ sizes of female mice that had been fed the ST or the LP diet for a period of 9 weeks

\begin{tabular}{lccc}
\hline & ST & LP & Student's $t$-test $(P$-value $)$ \\
\hline Body mass (g) & $22.7 \pm 0.5$ & $22.1 \pm 0.5$ & 0.41 \\
Heart WM (mg) & $119.7 \pm 5.0$ & $111.4 \pm 5.0$ & 0.26 \\
Liver WM (mg) & $940 \pm 40$ & $937 \pm 40$ & 0.95 \\
Kidneys WM (mg) & $257 \pm 7$ & $226 \pm 6$ & 0.004 \\
Ovary fat WM (mg) & $464 \pm 21$ & $521 \pm 21$ & 0.067 \\
Kidney fat WM (mg) & $93.5 \pm 7.8$ & $112.2 \pm 7.8$ & 0.11 \\
Kidney fat + ovary fat WM (mg) & $558 \pm 25$ & $633 \pm 25$ & 0.046 \\
Gut length (cm) & $39.7 \pm 0.7$ & $39.6 \pm 0.7$ & 0.90 \\
Small intestine length (cm) & $32.4 \pm 0.5$ & $33.4 \pm 0.5$ & 0.17 \\
Small intestine WM (mg) & $872 \pm 62$ & $991 \pm 72$ & 0.23 \\
Small intestine DM (mg) & $142.4 \pm 14.9$ & $166.3 \pm 17.2$ & 0.31 \\
Stomach DM (mg) & $36.4 \pm 0.8$ & $37.3 \pm 0.7$ & 0.36 \\
Colon + cecum DM (mg) & $62.6 \pm 3.8$ & $71.5 \pm 3.8$ & 0.13 \\
Total gut DM (mg) (intestine + cecum + colon) & $206 \pm 18$ & $234 \pm 20$ & 0.31 \\
Mucosa proportion (\% of total dry mass) & $71.8 \pm 2.3$ & $74.9 \pm 2.3$ & 0.36 \\
\hline
\end{tabular}

ST, standard diet; LP, low-protein diet; WM, wet mass; DM, dry mass.

All values presented as mean \pm S.E. ( $n=9$ in each group) and compared by Student's $t$-test. 

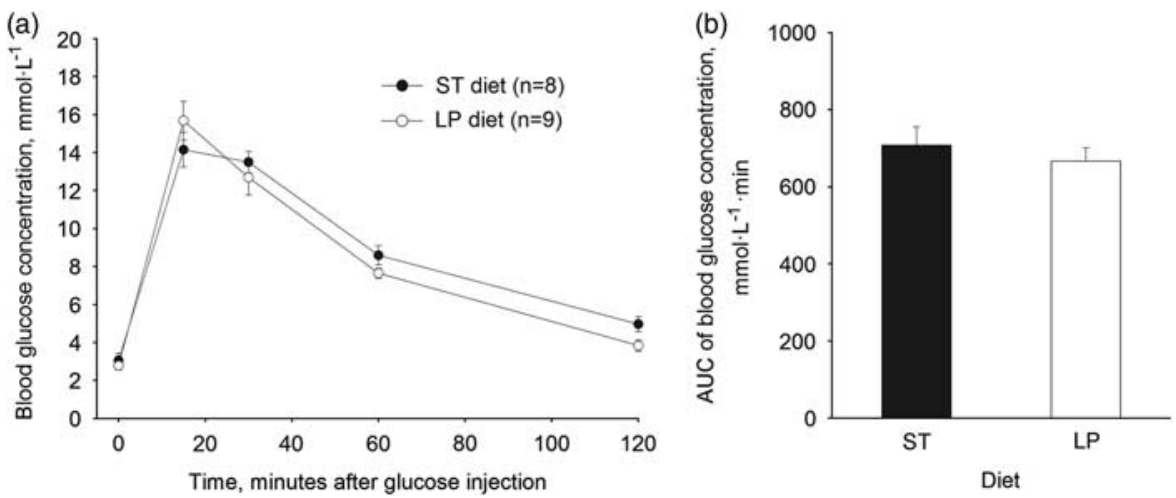

Fig. 2. Intraperitoneal glucose tolerance test (GTT) results. (a) Plasma glucose concentrations during GTT and (b) calculated Area Under the Curve (AUC). GTT was performed on female mice fed either the standard (ST; $n=9$ ) or the low-protein (LP; $n=9)$ diet for a period of 9 weeks (mean \pm S.E.). AUC values were compared using Student's $t$-test.

significant when corrected for body mass (least square means \pm s.E., $S T=260 \pm 5, \mathrm{LP}=230 \pm 5$, ANCOVA, body mass $F_{1}=35.46, P=0.0001$, diet: $\left.F_{1}=22.09, P=0.0003\right)$. Fat deposits were significantly larger in females fed the LP diet (Table 2), even after correcting for total body mass (least square means \pm s.E., $S T=551 \pm 23, \mathrm{LP}=640 \pm 23$, ANCOVA, body mass $F_{1}=4.4, P=0.053$, diet: $F_{1}=7.63$, $P=0.01)$. Visceral organ masses varied with body mass, but neither the absolute (Table 2) nor the body mass-corrected organ masses proved to be significantly different between the two treatments.

\section{Mating success}

From the 35 females that were mated (LP: $n=18$, ST: $n=17$ ), 10 females delivered offspring (LP: $n=6$, ST: $n=4$ ). The mean litter size in the LP group was $6 \pm 7$ (range 3-8 pups) and in the ST group 5.5 \pm 0.9 (range 4-7 pups). The mean body mass of LP females who delivered offspring was $18.8 \pm 0.5 \mathrm{~g}$ before and $21.4 \pm 0.6 \mathrm{~g}$ after 9 weeks of feeding on the LP diet $(n=6)$. The mean body mass of ST diet females who delivered offspring was $20.0 \pm 0.6 \mathrm{~g}$ before and $22.0 \pm 0.8$ after the dietary treatment $(n=4)$. In total, 51 pups were born: LP females delivered 31 pups (females: $n=23$, males: $n=8$ ) and ST females delivered 20 pups (females: $n=13$, males: $n=7$ ).

\section{Offspring growth and food intake}

Male offspring born to mothers exposed to the LP diet before conception had significantly lower body mass at weaning $(8.64 \pm 0.4 \mathrm{~g})$ than male offspring from the ST group $(10.16 \pm 0.4 \mathrm{~g} ; t$-test, $\mathrm{DF}=13, t=2.59, P=0.02)$. A similar tendency was observed for female offspring, but it was only marginally significant. LP female offspring weighed $8.88 \pm 0.2 \mathrm{~g}$ and ST female offspring had a body mass of $9.59 \pm 0.6 \mathrm{~g}$ ( $t$-test, $\mathrm{DF}=34, t=1.91, P=0.065)$.

A positive significant relationship was observed between the average male offspring body mass per litter at weaning and maternal body mass before conception (ANCOVA, maternal body mass before conception: $F_{1}=6.25, P=0.04$ ). A similar but nonsignificant tendency was observed for female offspring (ANCOVA, maternal body mass before conception: $F_{1}=4.56, P=0.06$ ). These differences in offspring body mass disappeared within 1 week after weaning (Fig. 3). The differences in body mass at weaning were not affected by litter size for either males (ANCOVA, litter size: $F_{1}=0.007$, $P=0.94$ ) or females (ANCOVA, litter size: $F_{1}=0.16$, $P=0.69)$.

Over the entire female offspring growth period, maternal diet and offspring age had significant effects on body mass; however, there was no interaction between the two (Fig. 3a; repeated measures MANOVA, maternal diet: $F_{1,33}=4.44, P=0.04$; offspring age: $F_{7,27}=254.6, P<0.0001$; maternal diet $\times$ time: $\left.F_{7,27}=0.95, P=0.48\right)$. Thus, growth curves $\operatorname{did}$ not have a significantly different pattern. Only offspring age had a significant effect on male offspring body mass, indicating that growth in body mass did occur during the experiment (Fig. 3b; repeated measures MANOVA, maternal diet: $F_{1,13}=3.23$, $P=0.1$; offspring age: $F_{7,7}=0.72, P<0.0001$; maternal diet $\times$ offspring age: $F_{7,7}=0.72, P=0.66$ ).

After the 6-week-long post-weaning period, female offspring total food consumption averaged at $197.1 \pm 4 \mathrm{~g}$ per individual in each group and there was no significant difference between ST and LP treatments ( $t$-test, $\mathrm{DF}=34, t=0.117, P=0.91)$. For the same period of time, male offspring total food intake averaged at $193.7 \pm 5 \mathrm{~g}$ per individual in both ST and LP groups ( $t$-test, $\mathrm{DF}=14, t=0.21, P=0.84$; Fig. 4 ).

\section{Offspring GTT and insulin}

GTT performed on adult offspring revealed that LP male offspring were more glucose tolerant than ST male offspring (Fig. $5 \mathrm{~d}$ and $5 \mathrm{e}, \mathrm{DF}=11, t$-test, $t=2.2, P=0.047$ ), but this was not observed for female offspring (Fig. 5a and 5b, $t$-test, $\mathrm{DF}=33, t=-0.06, P=0.948)$. However, LP female offspring had a significantly lower blood insulin concentration 

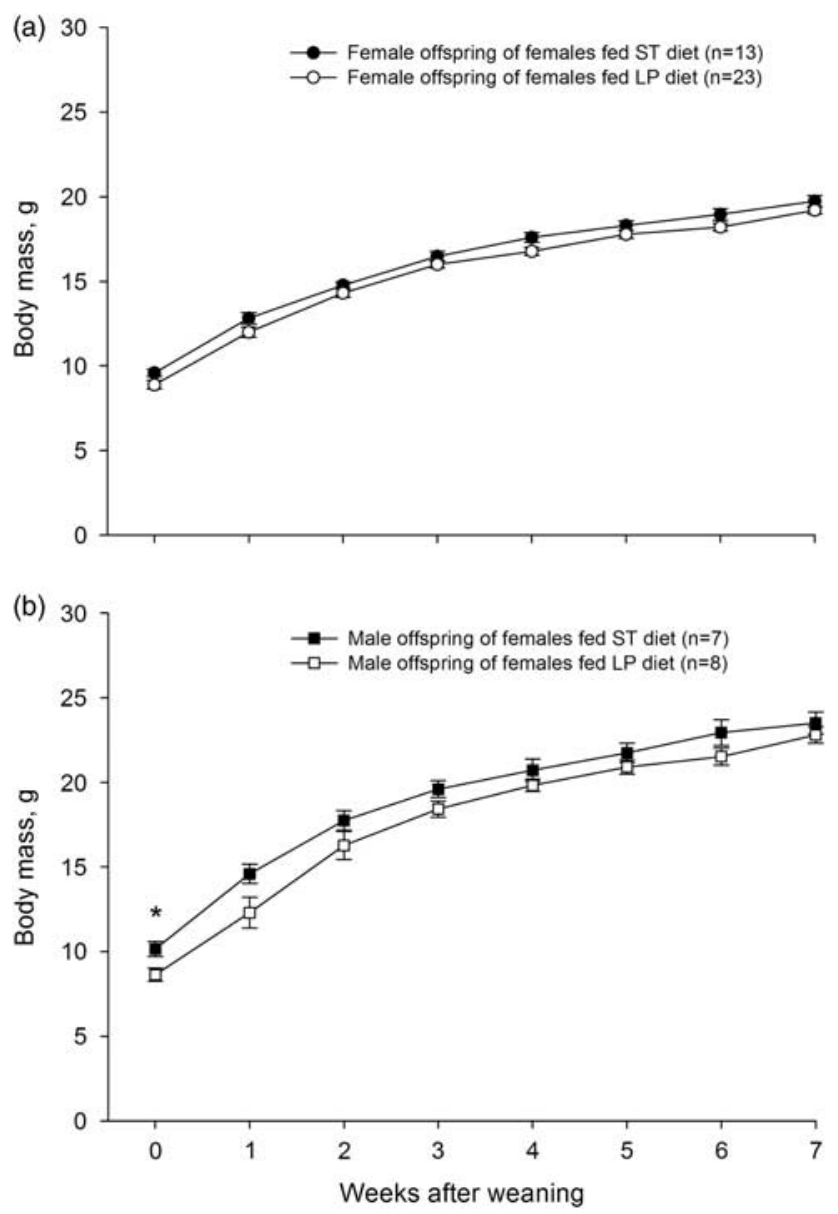

Fig. 3. Offspring body mass after weaning. Female (a) and male (b) offspring born to mothers fed the standard (ST) or the low-protein (LP) diet for 10 weeks before conception. Litter number, ST: $n=4$, LP: $n=6$. Body masses were compared by Student's $t$-test within the same gender for separate time points. ${ }^{*}$ Denotes a significant difference between the maternal dietary treatments, $P<0.05$.

than ST female offspring $2 \mathrm{~h}$ after glucose challenge (Fig. $5 \mathrm{c}$, $t$-test, $\mathrm{DF}=15, t=2.4, P=0.03)$. Such differences were not observed for male offspring (Fig. 5f, $t$-test, $\mathrm{DF}=7, t=1.7$, $P=0.13$ ). No differences were observed in male and female offspring fasting (Fig. $5 \mathrm{c}$ and $5 \mathrm{f}$, male offspring, $t$-test, $\mathrm{DF}=7$, $t=-0.93, \quad P=0.38 ;$ female offspring, $t$-test, $\quad \mathrm{DF}=26$, $t=0.94, P=0.35)$ and non-fasting insulin concentrations (male offspring, $\mathrm{DF}=12, t$-test, $t=-0.04, P=0.97$; female offspring, $t$-test, $\mathrm{DF}=30, t=1.02, P=0.32$ ).

\section{Offspring organ size}

We found no differences in offspring absolute and body mass-corrected visceral organ size (Tables 3 and 4). Stomach dry mass was significantly lower in LP female group (Table 3), but this difference disappeared when stomach dry mass was corrected for wet body mass (Table 4). Litter size had no significant effect on any of the measured values.
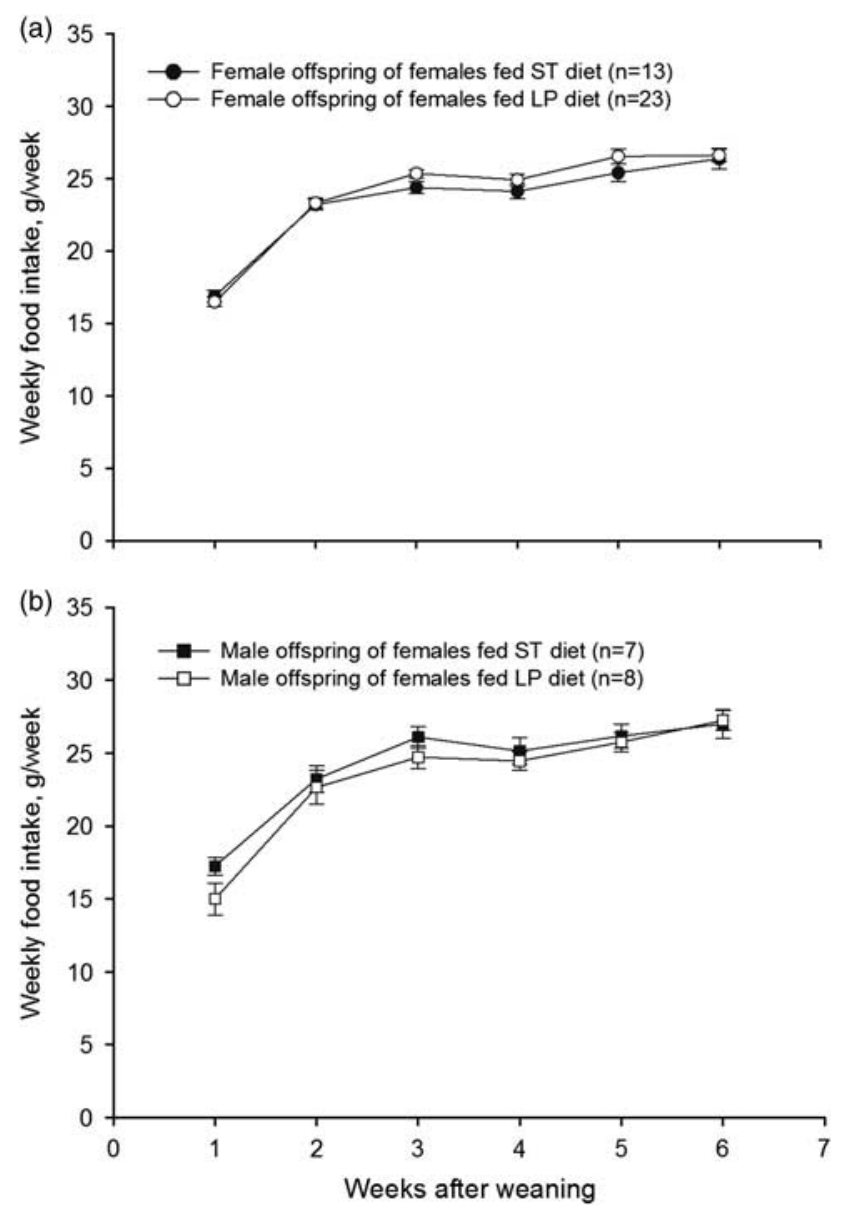

Fig. 4. Offspring food intake after weaning. Female $(a)$ and male (b) offspring born to mothers fed the standard (ST) or the low-protein (LP) diet for 10 weeks before conception. Litter number, ST: $n=4$, LP: $n=6$.

\section{Discussion}

Our study shows that protein restriction in the diet before conception may lower body mass at weaning, followed by catch-up growth and increased insulin sensitivity at early adulthood in mice. These effects are similar to those previously described for protein restriction during pregnancy and shown to be an indicator for increased risk of developing T2DM, CVD and obesity in humans and animals. ${ }^{6,7,9,20-27}$ It is therefore possible that LP diet before pregnancy imposes similar types of risks for the development of lifestyle diseases in the offspring as those described for malnutrition during pregnancy.

Offspring subjected to intrauterine growth restriction are often observed to display rapid postnatal growth if exposed to a nutrient-rich environment after birth. ${ }^{21,28}$ It is not clear whether catch-up growth is a risk factor that acts in addition to protein restriction when it comes to development of T2DM, CVD and obesity. However, in rodents, it appears to reduce offspring lifespan. ${ }^{28-30}$ In our study, male offspring 

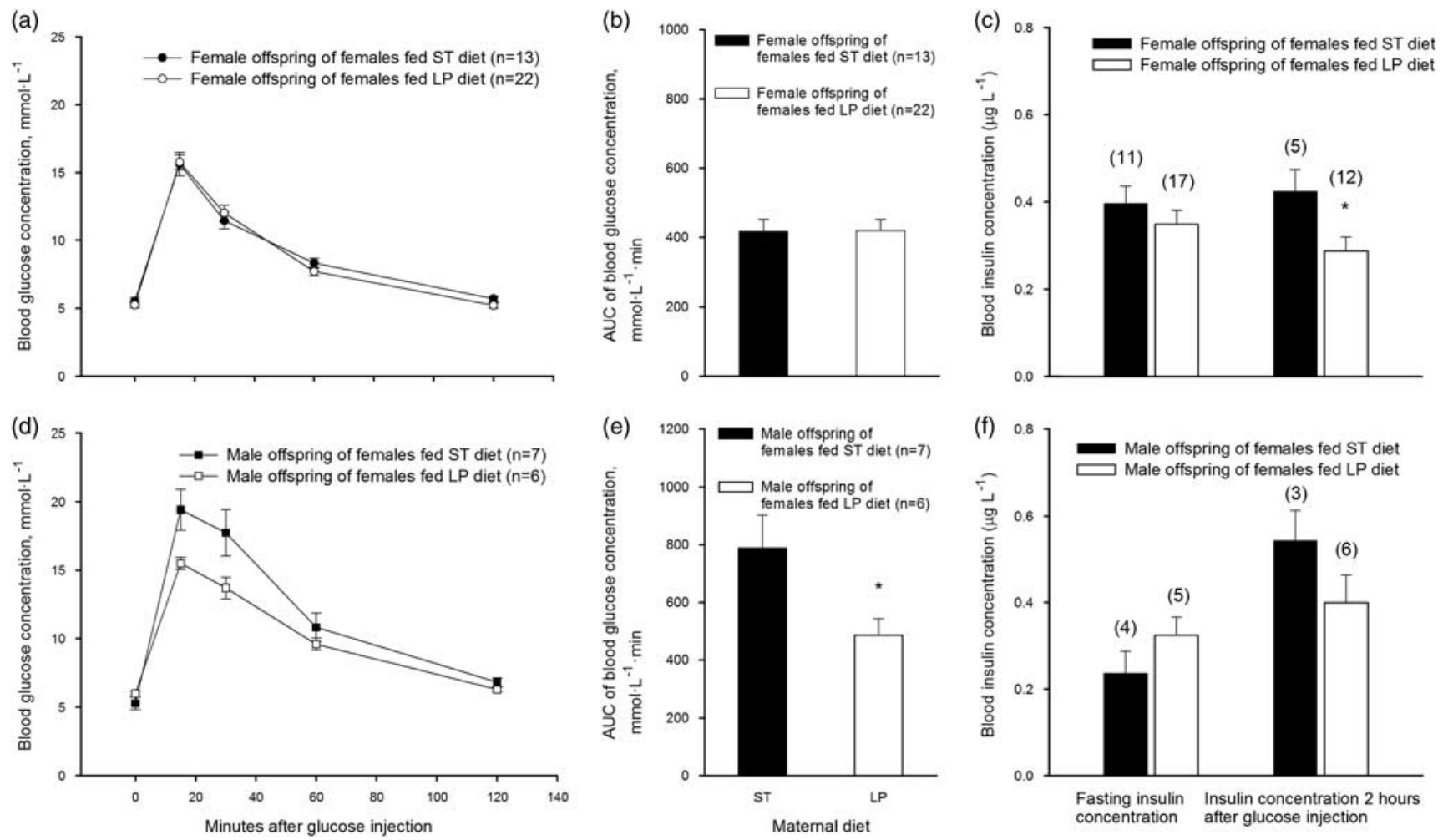

Fig. 5. Offspring glucose tolerance test (GTT) results and plasma insulin concentration. Blood glucose concentrations and Area Under the Curve (AUC) during GTT in female $(a, b$, respectively) and male $(d, e$, respectively) offspring born to mothers fed either the standard (ST) or the low-protein (LP) diet for 10 weeks before conception. Female $(c)$ and male $(f)$ offspring plasma insulin concentration after fasting and $2 \mathrm{~h}$ after glucose injection $(2 \mathrm{~g} / \mathrm{kg})$ during GTT. In $(c)$ and $(f)$, numbers in brackets indicate number of samples tested. Litter number, ST: $n=4$, LP: $n=6$. AUC and plasma insulin concentrations were compared by Student's $t$-test within the same gender.

${ }^{*}$ Denotes a significant difference between the maternal dietary treatments, $P<0.05$.

Table 3. Organ size of female and male offspring born to mothers fed either the ST or the LP diet for 10 weeks before conception

\begin{tabular}{|c|c|c|c|c|c|c|}
\hline & \multicolumn{3}{|c|}{ Female offspring } & \multicolumn{3}{|c|}{ Male offspring } \\
\hline & \multicolumn{2}{|c|}{ Maternal diet } & \multirow[b]{2}{*}{$P$} & \multicolumn{2}{|c|}{ Maternal diet } & \multirow[b]{2}{*}{$P$} \\
\hline & ST & LP & & ST & $\mathrm{LP}$ & \\
\hline Body mass at 11 weeks of age $(\mathrm{g})$ & $19.7 \pm 0.3$ & $19.2 \pm 0.2$ & 0.17 & $23.5 \pm 0.6$ & $22.8 \pm 0.6$ & 0.40 \\
\hline Heart WM (mg) & $111.6 \pm 3.4$ & $108.8 \pm 2.5$ & 0.51 & $136.5 \pm 4.4$ & $126.0 \pm 4.1$ & 0.10 \\
\hline Liver WM (mg) & $1026 \pm 30$ & $1028 \pm 22$ & 0.95 & $1263 \pm 39$ & $1266 \pm 37$ & 0.96 \\
\hline Kidney WM (mg) & $241 \pm 4$ & $244 \pm 3$ & 0.56 & $296 \pm 6$ & $288 \pm 6$ & 0.34 \\
\hline Kidney fat $(\mathrm{mg})$ & $55.6 \pm 4.8$ & $55.8 \pm 3.6$ & 0.98 & $69.7 \pm 4.9$ & $66.5 \pm 4.2$ & 0.63 \\
\hline Gut length $(\mathrm{cm})$ & $41.4 \pm 0.7$ & $41.8 \pm 0.5$ & 0.67 & $42.5 \pm 1.1$ & $41.3 \pm 1.0$ & 0.46 \\
\hline Small intestine length $(\mathrm{cm})$ & $32.2 \pm 0.5$ & $32.3 \pm 0.4$ & 0.91 & $34.3 \pm 1.0$ & $32.9 \pm 0.8$ & 0.28 \\
\hline Small intestine WM (mg) & $1074 \pm 37$ & $1017 \pm 29$ & 0.23 & $1106 \pm 45$ & $1071 \pm 39$ & 0.56 \\
\hline Small intestine DM (mg) & $193.2 \pm 7.6$ & $187.7 \pm 5.8$ & 0.57 & $201.7 \pm 8.9$ & $192.2 \pm 7.7$ & 0.44 \\
\hline Stomach DM (mg) & $32.5 \pm 0.8$ & $29.8 \pm 0.6$ & 0.011 & $31.6 \pm 1.1$ & $32.9 \pm 1.1$ & 0.45 \\
\hline Colon + cecum DM (mg) & $80.6 \pm 2.1$ & $79.4 \pm 1.6$ & 0.65 & $83.7 \pm 3.1$ & $79.8 \pm 2.9$ & 0.37 \\
\hline Total gut DM (mg) & $274 \pm 9$ & $267 \pm 7$ & 0.55 & $287 \pm 11$ & $272 \pm 9$ & 0.32 \\
\hline Mucosa proportion (\% of total dry mass) & $74.7 \pm 2.1$ & $77.1 \pm 1.6$ & 0.35 & $73.9 \pm 3.2$ & $80.0 \pm 3.0$ & 0.20 \\
\hline
\end{tabular}

ST, standard diet; LP, low-protein diet; WM, wet mass; DM, dry mass.

All values presented as mean \pm S.E. (female offspring, ST: $n=13$, LP: $n=23$; male offspring, ST: $n=7$, LP: $n=8$; litter number, ST: $n=4$, LP: $n=6$ ) and compared by Student's $t$-test. 
Table 4. Body mass-corrected organ size of female and male offspring born to mothers who were fed either the ST or the LP diet for 10 weeks before conception

\begin{tabular}{|c|c|c|c|c|c|c|c|c|}
\hline & \multicolumn{4}{|c|}{ Female offspring } & \multicolumn{4}{|c|}{ Male offspring } \\
\hline & \multicolumn{2}{|c|}{ Least square means \pm s.E. } & \multicolumn{2}{|c|}{ Effect tests ( $P$-value $)$} & \multicolumn{2}{|c|}{ Least square means \pm S.E. } & \multicolumn{2}{|c|}{ Effect tests ( $P$-value) } \\
\hline & ST & LP & Body mass & Maternal diet & ST & LP & Body mass & Maternal diet \\
\hline Heart WM (mg) & $109.3 \pm 2.9$ & $110.0 \pm 2.2$ & 0.0002 & 0.85 & $134.7 \pm 3.6$ & $127.5 \pm 3.3$ & 0.015 & 0.17 \\
\hline Liver WM (mg) & $998 \pm 16$ & $1037 \pm 12$ & $<0.0001$ & 0.07 & $1247 \pm 31$ & $1281 \pm 29$ & 0.01 & 0.45 \\
\hline Kidney WM (mg) & $238 \pm 4$ & $246 \pm 3$ & 0.0007 & 0.10 & $293 \pm 3$ & $291 \pm 3$ & $<0.0001$ & 0.68 \\
\hline Kidney fat (mg) & $54.9 \pm 4.9$ & $55.2 \pm 3.7$ & 0.42 & 0.95 & $54.9 \pm 4.9$ & $55.2 \pm 3.7$ & 0.42 & 0.96 \\
\hline Intestine WM (mg) & $1055 \pm 35$ & $1029 \pm 27$ & 0.01 & 0.57 & $1099 \pm 48$ & $1076 \pm 41$ & 0.62 & 0.73 \\
\hline Intestine DM (mg) & $189.8 \pm 7.3$ & $190.8 \pm 5.7$ & 0.03 & 0.92 & $200.1 \pm 9.5$ & $193.5 \pm 8.1$ & 0.54 & 0.62 \\
\hline Stomach DM (mg) & $31.1 \pm 0.9$ & $33.3 \pm 0.8$ & 0.006 & 0.10 & $31.1 \pm 0.9$ & $33.3 \pm 0.8$ & 0.006 & 0.097 \\
\hline Colon + cecum DM (mg) & $81.8 \pm 2.1$ & $79.8 \pm 1.5$ & 0.46 & 0.44 & $82.6 \pm 2.7$ & $80.7 \pm 2.5$ & 0.035 & 0.63 \\
\hline Total gut DM (mg) & $270 \pm 8$ & $271 \pm 7$ & 0.037 & 0.96 & $283 \pm 11$ & $275 \pm 9$ & 0.29 & 0.57 \\
\hline
\end{tabular}

ST, standard diet; LP, low-protein diet; WM, wet mass; DM, dry mass.

All values presented as least square mean \pm s.E. (female offspring, ST: $n=13$, LP: $n=23$; male offspring, ST: $n=7$, LP: $n=8$; litter number, ST: $n=4$, LP: $n=6$ ) of measures adjusted for wet body mass. In all analysis, litter size was used as a covariate first, but as it did not have a significant effect it was removed from the final model.

from the LP group had lower body mass at weaning than those from the ST group, but these differences disappeared within a few weeks after weaning (Fig. 3). This implies that the offspring have gone through a period of fast catch-up growth. Such catch-up growth has been shown in humans and several different animal species, ${ }^{24,31,32}$ including mice, ${ }^{33}$ and is associated with enhanced insulin sensitivity. ${ }^{34}$ This pattern corresponds well with our observed results of low male offspring early body mass, catch-up growth and enhanced insulin sensitivity in both sexes (Fig. 5) and indicates the presence of an event cascade similar to that induced by maternal gestational malnutrition.

In other animal models, similar offspring responses have been found. For example, rat offspring from mothers exposed to the LP diet during pregnancy also have increased insulin sensitivity at early adulthood (6-10 weeks), but this effect disappears with age (15 months), when offspring develop insulin resistance and glucose intolerance. ${ }^{22-24}$ As our findings from manipulating preconception nutrition resemble the cascade of events caused by malnutrition during pregnancy, it is possible that offspring born from mothers that received the LP diet before pregnancy are at the same increased risk of developing insulin resistance later in life. In a previous study with the same mouse strain and the same experimental protocol as in the current experiment, it was found that LP male offspring have increased adiposity as early as 46 days of age. ${ }^{17}$ The two studies in combination indicate that maternal protein restriction before pregnancy affects offspring body mass, body composition and glucose metabolism and can therefore modify offspring risk of developing lifestyleacquired diseases.
Although animal models using the LP diet after conception are rather well studied in terms of produced offspring phenotypes, the mechanisms underlying these effects are less clear. ${ }^{35}$ Some of the effects of LP diet exposure during pregnancy have been attributed to insufficient amount of amino acid supply to the embryo during development; ${ }^{9}$ however, our study and previous experiments ${ }^{17}$ have showed that similar effects can be induced by preconceptional protein restriction. This indicates that factors other than maternal diet during pregnancy can be involved in fetal programming. Unfortunately, our study design does not allow us to fully elucidate whether or not the risks are imposed through maternal body composition or physiological state, rather than by direct effects of amino acid insufficiency during development. Although studies exposing animals to protein restriction during pregnancy mostly ascribe the effects obtained to reduced amino acid supply, ${ }^{9}$ there is evidence that maternal body mass index, body composition, hypertension and diabetes among other factors can all have an impact on offspring health. ${ }^{36,37}$

Studies in human population show that maternal body mass index before pregnancy and weight gain during pregnancy are good predictors of offspring birth weight. ${ }^{12,38}$ Although we did not measure maternal weight gain during pregnancy to avoid stress to the pregnant females, ${ }^{39}$ we observed a significant relationship between maternal body mass at conception and male offspring body mass at weaning and a similar, nearly significant tendency for female offspring. It is likely that low body mass at weaning among LP offspring can be explained, at least partly, by low maternal body mass at conception, but these effects remain to be investigated. 
Studies on rodents pertaining to maternal obesity induced by a high-fat diet show that obesity at conception programs offspring obesity and insulin resistance at adulthood but do not necessarily affect birth weight. ${ }^{40}$ Our results showed that females fed the LP diet had significantly larger fat deposits at the time of conception than did females fed the ST diet (Table 2). It is difficult to discriminate whether increased adiposity in mothers fed the LP diet had an effect on offspring early body mass, but as suggested by previous studies it could have influenced their glucose metabolism. ${ }^{41}$

Another aspect of offspring programming is maternal insulin-mediated glucose metabolism. Elevated maternal blood glucose differentially affects birth weight: whereas mild elevation above normoglycemia produces macrosomic offspring, highly elevated glucose levels induce fetal growth restriction. ${ }^{42}$ GTT on females at the end of preconceptional feeding period showed no differences in fasting glucose values or glucose tolerance (Fig. 2). However, non-fasting blood insulin concentration was nearly significantly lower in LP-fed dams, a known result of LP feeding. ${ }^{43,44}$ As exposure of preimplantation murine embryos to insulin increases fetal growth rate in vitro, ${ }^{45}$ it is possible that lowered maternal insulin levels in the LP group at the time of conception have restricted offspring growth.

Whereas our study mainly addresses the physiological changes of the mother that can affect offspring development and phenotype, other studies have shown that similar effects in offspring can be induced without apparent changes in maternal phenotype. Thus, female mice of the MF-1 strain fed an LP diet for a very short period of time close to conception ( \pm 3.5 days, which includes the period of oocyte maturation and preimplantation) produced offspring that at 21 and 52 weeks of age had elevated systolic blood pressure $e^{16,46}$ and attenuated endothelium-dependent vasodilation in mesenteric arteries at the age of 22 weeks $^{16}$ compared with control offspring whose mothers were supplied with sufficient protein. As such short feeding periods may be expected to have only minor effects on the body composition of the mothers, compared with the 10-week period of feeding in our experiment, the measured alterations in the MF-1 offspring could very well have been caused by epigenetic changes in the mothers eggs or in the young zygote. It is therefore of high importance to investigate such epigenetic effects in the future studies on the presented animal model of preconceptional nutrition.

Other studies have found that maternal LP nutrition during pregnancy can program offspring food intake and preferences, ${ }^{47}$ which can potentially explain differences in offspring growth, development of obesity, etc. In our study, we measured offspring food intake throughout the experiment and found no differences. Thus, we find no evidence that differences observed in offspring phenotypes were caused by differences in their feeding behavior after weaning.

Fertility rates observed in the study were rather low. Such low conception rates may be explained by the use of suboptimal mating protocol. Estrous cycle frequency in mice is 4-5 days, but the females were only caged with the males for 2 days, which may explain the low rate of conception. For the future studies, females and males should be housed together for a longer period ( $4-5$ days). The study would have had greater statistical power if the litter numbers had been higher; however, the data presented were tested using appropriate statistical tests, and the effects of maternal preconceptional treatment are evident. It is, however, possible that because of low litter numbers some of the effects could not have not been detected, and therefore future studies need to be carried out, where another mating protocol is used and higher litter numbers are obtained.

As suggested by previous epidemiological studies, maternal preconceptional nutrition and physiological state can have an impact on the future health of the offspring in humans. ${ }^{11,12}$ Our studies using an animal model of preconceptional nutrition ${ }^{17}$ confirm the importance of these effects. It is therefore important to investigate the effects of maternal preconceptional state further, as, once established, they can provide effective grounds for intervention and prevention of disease development in future generations of human populations.

Overall, our findings show that maternal protein restriction before pregnancy induces gender-specific effects in the offspring, namely, reduced early body mass, male offspring catch-up growth and enhanced insulin sensitivity at early adulthood in male and female offspring. These effects closely resemble the effects of maternal protein restriction during pregnancy on offspring physiology and may therefore imply an increased risk for the offspring to develop obesity, type II diabetes and CVD in later life.

The physiological mechanisms inducing these changes are less clear and remain to be investigated.

\section{Acknowledgments}

D.M. and T.W. were funded by the grants from Danish Research Council. G.W. was funded by Danish Medical Research Council, grant no. 271-08-0768. We thank Heidi Meldgaard Jensen and Rasmus Buchanan for their assistance during experiments.

\section{References}

1. World Health Organization (WHO). Global status report on noncommunicable diseases. In Description of the Global Burden of NCDs, their Risk Factors and Determinants (ed. Ala Alwan), 2010. WHO: Geneva.

2. Yang WJ, Kelly T, He J. Genetic epidemiology of obesity. Epidemiol Rev. 2007; 29, 49-61.

3. Kunz LH, King JC. Impact of maternal nutrition and metabolism on health of the offspring. Semin Fetal Neonatal Med. 2007; 12, 71-77.

4. Warner MJ, Ozanne SE. Mechanisms involved in the developmental programming of adulthood disease. Biochem J. 2010; 427, 333-347. 
5. Barker DJP. The fetal and infant origins of adult disease. Br Med J. 1990; 301, 1111.

6. Barker DJ. The intrauterine origins of cardiovascular and obstructive lung disease in adult life. The Marc Daniels Lecture 1990. J R Coll Physicians Lond. 1991; 25, 129-133.

7. Hales C, Barker D, Clarc P, et al. Fetal and infant growth and impaired glucose tolerance at age 64 years. Br Med J. 1991; 303, 1019-1022.

8. Hales CN, Barker DJ. Type 2 (non-insulin-dependent) diabetes mellitus: the thrifty phenotype hypothesis. Diabetologia. 1992; 35, 595-601.

9. Bhasin KKS, van Nas A, Martin LJ, et al. Maternal low-protein diet or hypercholesterolemia reduces circulating essential amino acids and leads to intrauterine growth restriction. Diabetes. 2009; 58, 559-566.

10. Vickers MH, Breier BH, Cutfield WS, Hofman PL, Gluckman PD. Fetal origins of hyperphagia, obesity, and hypertension and postnatal amplification by hypercaloric nutrition. Am J Physiol-Endoc M. 2000; 279, E83-E87.

11. Cuco G, Arija V, Iranzo R, et al. Association of maternal protein intake before conception and throughout pregnancy with birth weight. Acta Obstet Gyn Scan. 2006; 85, 413-421.

12. Weisman CS, Misra DP, Hillemeier MM, et al. Preconception predictors of birth outcomes: prospective findings from the Central Pennsylvania Women's Health Study. Matern Child Health J. 2011; 15, 829-835.

13. Barker DJ, Winter PD, Osmond C, Margetts B, Simmonds SJ. Weight in infancy and death from ischaemic heart disease. Lancet. 1989; 2, 577-580.

14. Harder T, Rodekamp E, Schellong K, Dudenhausen JW, Plagemann A. Birth weight and subsequent risk of type 2 diabetes: a meta-analysis. Am J Epidemiol. 2007; 165, 849-857.

15. Torrens C, Snelling TH, Chau R, et al. Effects of pre- and periconceptional undernutrition on arterial function in adult female sheep are vascular bed dependent. Exp Physiol. 2009; 94, 1024-1033.

16. Watkins AJ, Wilkins A, Cunningham C, et al. Low protein diet fed exclusively during mouse oocyte maturation leads to behavioural and cardiovascular abnormalities in offspring. J Physiol (Lond). 2008; 586, 2231-2244.

17. Mortensen EL, Wang T, Malte H, Raubenheimer D, Mayntz D. Maternal preconceptional nutrition leads to variable fat deposition and gut dimensions of adult offspring mice (C57BL/6JBom). Int J Obes (Lond). 2010; 34, 1618-1624.

18. Sorensen A, Mayntz D, Raubenheimer D, Simpson SJ. Proteinleverage in mice: the geometry of macronutrient balancing and consequences for fat deposition. Obesity. 2008; 16, 566-571.

19. Jensen AR, Elnif J, Burrin DG, Sangild PT. Development of intestinal immunoglobulin absorption and enzyme activities in neonatal pigs is diet dependent. J Nutr. 2001; 131, 3259-3265.

20. Baird J, Fisher D, Lucas P, et al. Being big or growing fast: systematic review of size and growth in infancy and later obesity. Br Med J. 2005; 331, 929-931.

21. Bieswal F, Ahn MT, Reusens B, et al. The importance of catch-up growth after early malnutrition for the programming of obesity in male rat. Obesity. 2006; 14, 1330-1343.

22. Hales C, Desai M, Ozanne S, Crowther N. Fishing in the stream of diabetes: from measuring insulin to the control of fetal organogenesis. Biochem Soc Trans. 1996; 24, 341-350.
23. Petry CJ, Dorling MW, Pawlak DB, Ozanne SE, Hales CN. Diabetes in old male offspring of rat dams fed a reduced protein diet. Int J Exp Diabetes Res. 2001; 2, 139-143.

24. Shepherd PR, Crowther NJ, Desai M, Hales CN, Ozanne SE. Altered adipocyte properties in the offspring of protein malnourished rats. Br J Nutr. 1997; 78, 121-129.

25. Woods LL, Weeks DA, Rasch R. Programming of adult blood pressure by maternal protein restriction: role of nephrogenesis. Kidney Int. 2004; 65, 1339-1348.

26. Yliharsila H, Kajantie E, Osmond C, et al. Birth size, adult body composition and muscle strength in later life. Int J Obes (Lond). 2007; 31, 1392-1399.

27. Zambrano E, Bautista CJ, Deas M, et al. A low maternal protein diet during pregnancy and lactation has sex- and window of exposure-specific effects on offspring growth and food intake, glucose metabolism and serum leptin in the rat. J Physiol (Lond). 2006; 571, 221-230.

28. Chen JH, Martin-Gronert MS, Tarry-Adkins J, Ozanne SE. Maternal protein restriction affects postnatal growth and the expression of key proteins involved in lifespan regulation in mice. PLoS One. 2009; 4, e4950.

29. Barnes SK, Ozanne SE. Pathways linking the early environment to long-term health and lifespan. Prog Biophys Mol Biol. 2011; 106, 323-336.

30. Jennings BJ, Ozanne SE, Dorling MW, Hales CN. Early growth determines longevity in male rats and may be related to telomere shortening in the kidney. Febs Letters. 1999; 448, 4-8.

31. De Blasio MJ, Gatford KL, McMillen IC, Robinson JS, Owens JA. Placental restriction of fetal growth increases insulin action, growth, and adiposity in the young lamb. Endocrinology. 2007; 148, 1350-1358.

32. Dulloo AG, Jacquet J, Seydoux J, Montani JP. The thrifty 'catch-up fat' phenotype: its impact on insulin sensitivity during growth trajectories to obesity and metabolic syndrome. Int J Obes. 2006; 30, S23-S35.

33. Cottrell EC, Martin-Gronert MS, Fernandez-Twinn DS, et al. Leptin-independent programming of adult body weight and adiposity in mice. Endocrinology. 2011; 152, 476-482.

34. Morrison JL, Duffield JA, Muhlhausler BS, Gentili S, McMillen IC. Fetal growth restriction, catch-up growth and the early origins of insulin resistance and visceral obesity. Pediatr Nephrol. 2010; 25, 669-677.

35. Rosario FJ, Jansson N, Kanai Y, et al. Maternal protein restriction in the rat inhibits placental insulin, mTOR, and STAT3 signaling and down-regulates placental amino acid transporters. Endocrinology. 2011; 152, 1119-1129.

36. de Bernabe JV, Soriano T, Albaladejo R, et al. Risk factors for low birth weight: a review. Eur J Obstet Gyn R B. 2004; $116,3-15$.

37. Ehrenberg HM, Mercer BM, Catalano PM. The influence of obesity and diabetes on the prevalence of macrosomia. Am J Obstet Gynecol. 2004; 191, 964-968.

38. Ehrenberg HM, Dierker L, Milluzzi C, Mercer BM. Low maternal weight, failure to thrive in pregnancy, and adverse pregnancy outcomes. Am J Obstet Gynecol. 2003; 189, 1726-1730.

39. Decatanzaro D, Macniven E. Psychogenic pregnancy disruptions in mammals. Neurosci Biobehav R. 1992; 16, 43-53. 
40. Shankar K, Harrell A, Liu XL, et al. Maternal obesity at conception programs obesity in the offspring. Am J Physiol-Reg I. 2008; 294, R528-R538.

41. Howie GJ, Sloboda DM, Kamal T, Vickers MH. Maternal nutritional history predicts obesity in adult offspring independent of postnatal diet. J Physiol. 2009; 587, 905-915.

42. Oh W, Gelardi NL, Cha CJ. Maternal hyperglycemia in pregnant rats - its effect on growth and carbohydratemetabolism in the offspring. Metab Clin Exp. 1988; 37, 1146-1151.

43. Jansson N, Pettersson J, Haafiz A, et al. Down-regulation of placental transport of amino acids precedes the development of intrauterine growth restriction in rats fed a low protein diet. J Physiol (Lond). 2006; 576, 935-946.
44. Kwong WY, Wild AE, Roberts P, Willis AC, Fleming TP. Maternal undernutrition during the preimplantation period of rat development causes blastocyst abnormalities and programming of postnatal hypertension. Development. 2000; 127, 4195-4202.

45. Kaye PL, Gardner HG. Preimplantation access to maternal insulin and albumin increases fetal growth rate in mice. Hum Reprod. 1999; 14, 3052-3059.

46. Watkins AJ, Lucas ES, Wilkins A, Cagampang FRA, Fleming TP. Maternal periconceptional and gestational low protein diet affects mouse offspring growth, cardiovascular and adipose phenotype at 1 year of age. PLoS One. 2011; 6, e28745.

47. Bellinger L, Lilley C, Langley-Evans SC. Prenatal exposure to a maternal low-protein diet programmes a preference for high-fat foods in the young adult rat. Br J Nutr. 2004; 92, 513-520. 